\title{
Coding of Non-coding RNA: Insights Into the Regulatory Functions of Pri-MicroRNA-Encoded Peptides in Plants
}

OPEN ACCESS

Edited by:

Cao Xu,

Chinese Academy of Sciences, China

Reviewed by:

Hongliang Zhu,

China Agricultural University, China

Xu Fang,

Shandong University, China

*Correspondence:

Chao Ma

chaoma2015@sjtu.edu.cn

Specialty section:

This article was submitted to

Plant Physiology,

a section of the journal

Frontiers in Plant Science

Received: 14 December 2020

Accepted: 25 January 2021

Published: 25 February 2021

Citation:

Ren $Y$, Song $Y$, Zhang $L$, Guo $D$,

He J, Wang L, Song S, Xu W,

Zhang C, Lers A, Ma C and Wang S

(2021) Coding of Non-coding RNA: Insights Into the Regulatory Functions

of Pri-MicroRNA-Encoded Peptides

in Plants. Front. Plant Sci. 12:641351.

doi: 10.3389/fpls.2021.641351
Yi Ren', Yue Song ${ }^{1}$, Lipeng Zhang ${ }^{2}$, Dinghan Guo ${ }^{2}$, Juan He ${ }^{1}$, Lei Wang ${ }^{1}$, Shiren Song ${ }^{1}$, Wenping $\mathrm{Xu}^{1}$, Caixi Zhang ${ }^{1}$, Amnon Lers ${ }^{3}$, Chao $\mathrm{Ma}^{1 *}$ and Shiping Wang ${ }^{1,4}$

\begin{abstract}
${ }^{1}$ Department of Plant Science, School of Agriculture and Biology, Shanghai Jiao Tong University, Shanghai, China, ${ }^{2}$ Department of Horticulture, College of Agriculture, Shihezi University, Shihezi, China, ${ }^{3}$ Department of Postharvest Science of Fresh Produce, Volcani Center, Agricultural Research Organization, Bet Dagan, Israel, ${ }^{4}$ Key Laboratory of Agro-products Processing Technology of Shandong, Institute of Agro-food Science and Technology, Shandong Academy of Agricultural Sciences, Jinan, China
\end{abstract}

Peptides composed of a short chain of amino acids can play significant roles in plant growth, development, and stress responses. Most of these functional peptides are derived by either processing precursor proteins or direct translation of small open reading frames present in the genome and sometimes located in the untranslated region sequence of a messenger RNA. Generally, canonical peptides serve as local signal molecules mediating short- or long-distance intercellular communication. Also, they are commonly used as ligands perceived by an associated receptor, triggering cellular signaling transduction. In recent years, increasing pieces of evidence from studies in both plants and animals have revealed that peptides are also encoded by RNAs currently defined as non-coding RNAs (ncRNAs), including long ncRNAs, circular RNAs, and primary microRNAs. Primary microRNAs (miRNAs) have been reported to encode regulatory peptides in Arabidopsis, grapevine, soybean, and Medicago, called miRNAencoded peptides (miPEPs). Remarkably, overexpression or exogenous applications of miPEPs specifically increase the expression level of their corresponding miRNAs by enhancing the transcription of the MIRNA (MIR) genes. Here, we first outline the current knowledge regarding the coding of putative ncRNAs. Notably, we review in detail the limited studies available regarding the translation of miPEPs and their relevant regulatory mechanisms. Furthermore, we discuss the potential cellular and molecular mechanisms in which miPEPs might be involved in plants and raise problems that needed to be solved.

Keywords: miPEP, miRNA-encoded peptide, miRNA, pri-miRNA, non-coding RNA, peptides

\section{INTRODUCTION}

For a long time, canonical phytohormones, such as auxin and cytokinin, offer the main perspective in our understanding of regulatory networks modulating plant growth, development, and stress response (Dubois et al., 2018; Kieber and Schaller, 2018). In the last decades, an increasing number of studies have focused on the central role of small peptides, called peptidehormones, as short- or long-distance signaling molecules to integrate internal cues with external 
environment stimuli (Huffaker et al., 2011; Ohkubo et al., 2017; Nakaminami et al., 2018; Olsson et al., 2019). Peptides are typically composed of 2 to 100 amino acid residues (Tavormina et al., 2015) and are commonly secreted into the apoplast. Known peptides usually act as ligands that bind to their receptors to activate downstream signaling cascades involved in plant innate immunity (Huffaker et al., 2006; Yamaguchi et al., 2006; Huffaker and Ryan, 2007), nutrient homeostasis (Okamoto et al., 2016), reproduction process (Kachroo et al., 2001; Okuda et al., 2009; Qu et al., 2015), stress response (Nakaminami et al., 2018; Takahashi et al., 2018), and morphogenesis (Yamaguchi et al., 2016; Qian et al., 2018). Although the majority of the reported functional peptides are derived from the processing of precursor proteins or the coding of small open reading frames (sORFs), numerous pieces of evidence from plants and animals have suggested that previously annotated noncoding RNAs (ncRNAs) may encode peptides, expanding the peptidome complexity (Ruiz-Orera et al., 2014; Lauressergues et al., 2015; Legnini et al., 2017). Primary microRNAs (primiRNAs), which are transcribed from the MIR genes and subsequently processed to produce the mature miRNAs, can actually encode regulatory miRNA-encoded peptides (miPEPs) in plants (Wang L. and Wang, 2015; Lv et al., 2016; Julkowska, 2020). According to available results, overexpression or external application of miPEPs can positively regulate the mature miRNAs by enhancing the transcription of their associated MIR genes, which is similar to the innate immunity system in plants where the endogenous peptides can also increase expression of their encoding precursor genes (Huffaker and Ryan, 2007; Huffaker et al., 2011; Couzigou et al., 2016). In this review, we review the coding of ncRNA, emphatically, focusing on the translation of pri-miRNA and their relevant biological functions and possible regulatory mechanisms.

\section{PEPTIDOME COMPLEXITY IN PLANTS}

Since systemin was first characterized in tomato, plant peptides are emerging as significant signaling molecules involved in different physiological processes (Pearce et al., 1991). They are categorized into precursor-derived peptides and nonprecursor-derived peptides based on their biogenesis (Figure 1). The precursor-derived peptides are further classified into post-translationally modified peptides, cysteine-rich peptides (CRPs), and peptides without rich cysteine or post-translational modification (Figure 1A). The non-precursor-derived peptides are mainly encoded by sORFs hidden in the plant genome, additional short ORFs in messenger RNA (mRNA) transcripts, and so-called ncRNAs (Figures 1B,C; Hanada et al., 2013; Tavormina et al., 2015; Oh et al., 2018).

\section{Peptides Encoded by Conventional Open Reading Frames}

The origins, functions, and functional mechanisms of peptides encoded by conventional ORFs in plants have been wellreviewed (Tavormina et al., 2015; Olsson et al., 2019). Generally, mature precursor-derived peptides are initially translated into larger non-functional prepropeptides and further processed by proteolytic cleavage and modification such as tyrosine (Tyr) sulfation, proline hydroxylation, and hydroxyproline arabinosylation, to yield biologically active peptides (Figure 1A; Matsubayashi, 2014; Olsson et al., 2019). The second precursorderived peptides are the CRPs characterized by a domain with 2-16 cysteine residues (Matsubayashi, 2014). CRPs are also processed, and the typical intramolecular disulfide bonds are catalyzed by protein disulfide isomerases (Figure 1A; Tavormina et al., 2015; Olsson et al., 2019). The third group of peptides processed from the non-functional precursors is named "non-Cys-rich/non-modification peptides", which contain several important amino acid residues such as proline, Gly, and lysine critical for biological activity (Figure 1A; Tavormina et al., 2015). Additionally, most gene annotation algorithms do not effectively distinguish between coding and non-coding sequences when the coding sequences are small. Therefore, thousands of SORFs are failed to be annotated in the plant genome as coding for proteins (Andrews and Rothnagel, 2014; Takahashi et al., 2019). In Arabidopsis, 8,000 putative sORFs with high coding potential are identified, of which $\sim 10 \%$ of identified peptides have a function based on the visible phenotypic effects revealed after their overexpression (Hanada et al., 2013). Therefore, it is a reasonable assumption that many functional sORFs are hidden in the plant genome (Hsu and Benfey, 2018; Figure 1B). In general, canonical peptides are thought to be phytohormonelike signaling molecules that mediate short- or long-distance intercellular communication and play an important role in regulating growth and development in plants (Ohkubo et al., 2017; Olsson et al., 2019).

\section{Coding of Short Open Reading Frames in Putative Non-coding RNAs}

In eukaryotic mRNA, one or more short ORFs may exist in $5 \times$ leader sequence [or $5^{\prime}$ untranslated region (UTR)] located in the upstream of the main protein-coding ORF, called upstream open reading frame (uORF) (Chen J. et al., 2020; Kurihara, 2020; Figure 1C). The uORF presumably serves as a post-transcriptional cis-regulatory element that represses the transcription of main protein-coding ORF by causing ribosome stalling and nonsense-mediated decay (von Arnim et al., 2014). In one case, vitamin $\mathrm{C} /$ ascorbate content is determined by the GDP-L-galactose phosphorylase (GGP) enzyme. An uORF located in the upstream UTR of the GGP gene, encoding 60-a.a. length peptide, serves as a cis-acting element that represses the translation of the downstream GGP ORF under high ascorbate concentration (Laing et al., 2015). Editing the uORF of GGP increases the vitamin C content by $\sim 150 \%$ (Zhang et al., 2018). In Arabidopsis, AtHB1 belongs to the homeodomainleucine zipper transcription factor family. The translation of $A t H B 1$ is post-transcriptionally repressed by the uORF located in the upstream of 5' UTR of AtHB1 through a ribosome stalling mechanism. This uORF encodes a conserved peptide in flowering plants, called CPuORF (Ribone et al., 2017; van der Horst et al., 2019). In addition to uORF in 5' UTR, 

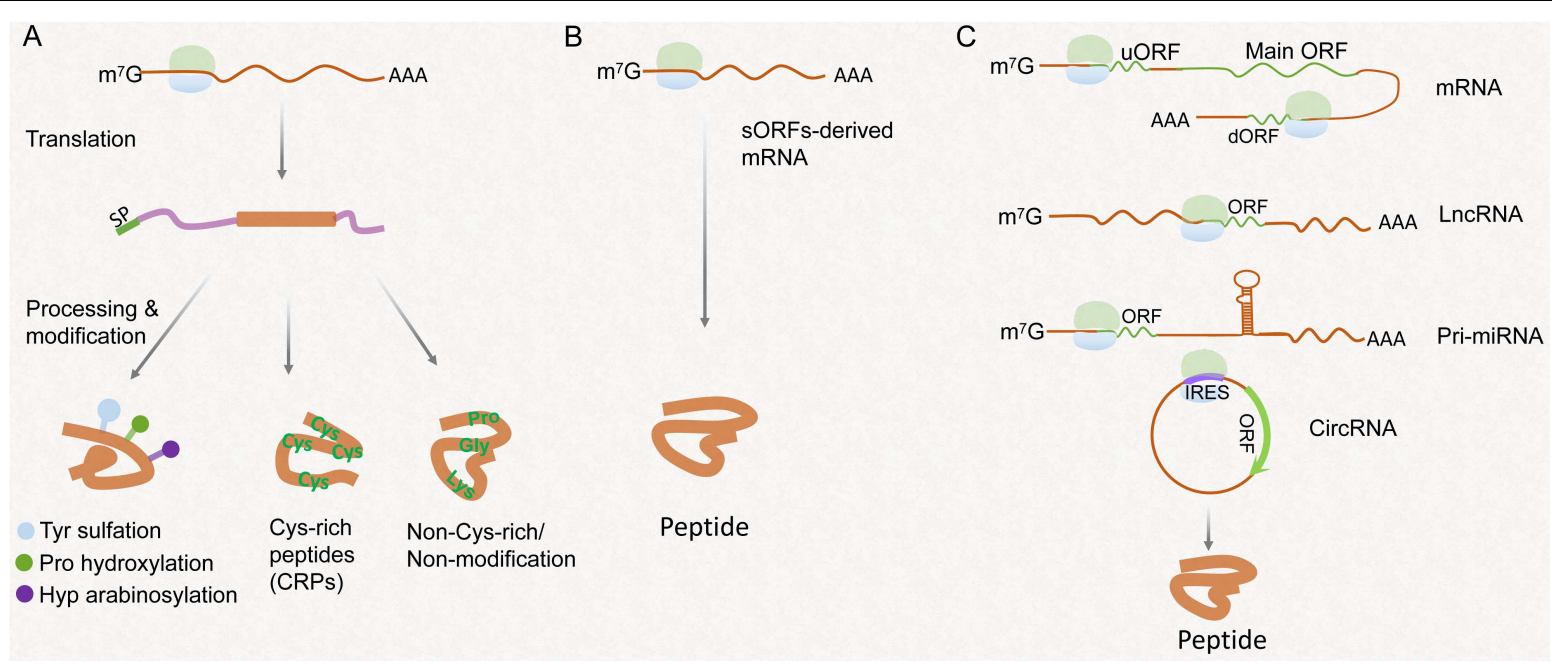

FIGURE 1 | Schematic presentation of the different pathways of peptide synthesis and processing. (A) Peptides processed from the precursor containing signaling peptide (SP). Modifications of peptides contain Tyr sulfation, proline hydroxylation, and hydroxyproline arabinosylation. Cys-rich peptides usually contain 2-16 Cys residues. Non-Cys-rich/non-modificational peptides contain several key residues such as proline, Gly, and lysine responsible for biological activity. (B) Peptides encoded by sORFs in genome. (C) Peptides encoded by an upstream ORF (uORF) or downstream ORF (dORF) of a protein-coding region, non-coding RNAs, including IncRNAs, pri-miRNAs, and circRNAs.

hundreds of sORFs have been identified in 3' UTR, called downstream UTRs (dUTRs), by ribosome profiling sequencing and proteomics analyses in mammalian cell (Chen J. et al., 2020; Wu et al., 2020). Contrary to uORFs, dUTRs were described to enhance the translation of their corresponding main ORF (Wu et al., 2020). Whether translation of dUTRs occurs in plants remains to be shown. Although the translation of sORFs derived from the 5' or 3' UTR of mRNA has been investigated, the biological functions of such peptides are not fully understood yet.

Ribosome profiling sequencing provides a feasible method to explore the coding potential of putative ncRNAs such as long non-coding RNAs (lncRNAs), circular RNAs (circRNAs), and pri-miRNA, although this cue is not sufficient to classify transcripts as coding or non-coding (Ingolia et al., 2012; Guttman et al., 2013). Recently, ncEP, a manually curated database for collecting validated ncRNA-encoded proteins or peptides, is constructed and enriches the repository of coding RNAs (Liu et al., 2020). LncRNAs are usually defined as transcripts that are longer than $200 \mathrm{nt}$ in length and do not encode a discernable protein (Chekanova, 2015). Like the mRNA, lncRNAs are transcribed by Pol II, capped in their 5' termini and polyadenylated in their 3' termini, and are accumulated in the cytoplasm (van Heesch et al., 2014). Ribosome profiling analyses of six species, including Arabidopsis, revealed that a large fraction of the lncRNAs are associated with ribosome protection (RuizOrera et al., 2014). However, most of the known peptides translated from lncRNA were mostly investigated in an animal cell such as HOXB-AS3, a conserved 53-a.a. peptide encoded by lncRNA $H O X B-A S 3$ that could suppress colon cancer growth (Huang et al., 2017). In Arabidopsis, the POLARIS (PLS) gene encoding a predicted peptide of 36-a.a. residues induced by auxin was located at a 500-nt position of this transcript. PLS is required to regulate auxin-cytokinin homeostasis for modulating root growth and leaf vascular patterning (Casson et al., 2002). In legumes, ENOD40 is expressed in root nodule organogenesis. Unlike canonical mRNA, ENOD40 is polycistronic RNA that encodes two ORFs and generates two small peptides with 12- and 24-a.a residues responsible for binding to sucrose synthase (Röhrig et al., 2002). These examples suggest that the presence of ORFs encoding for peptides in known lncORFs exists in plants; however, conclusive pieces of evidence require further investigation.

Circular RNAs (circRNAs) are produced by precursor mRNAs back-splicing where a downstream 5' splice site is covalently linked to an upstream 3' splice site in eukaryote (Conn et al., 2017; Li et al., 2018). In grapevine, approximately $91 \%$ of circulation events of circRNAs are exon-circulation (Gao et al., 2019), implying that circRNAs may function as a template to direct protein synthesis. In fact, the translation of some circRNAs has been discovered in animals, and the encoded peptides were found to control cell proliferation and play biological roles in disease response (Legnini et al., 2017; Shi et al., 2020). The absence of $\mathrm{m}^{7} \mathrm{GpppN}$ caps at the 5' end in circRNAs and lack of poly (A) tails at the 3' end cause cap-independent translation initiation in circRNAs (Diallo et al., 2019). Furthermore, if the circRNAs contain internal ribosome entry sites, the eukaryotic initiation factor (elF4G2) directly binds to the internal ribosome entry site and recruitments $43 \mathrm{~S}$ pre-initiation complex to initiate translation (Wang Y. and Wang, 2015; Diallo et al., 2019; Shi et al., 2020). In humans, the $\mathrm{N}^{6}$-methyladenosine $\left(\mathrm{m}^{6} \mathrm{~A}\right)$ of circRNAs drives the efficient initiation of protein translation, and even an $\mathrm{m}^{6} \mathrm{~A}$ motif, "RRm ${ }^{6} \mathrm{ACH}$ " ( $\mathrm{R}=\mathrm{G}$ or $\mathrm{A} ; \mathrm{H}=\mathrm{A}, \mathrm{C}$ or $\left.\mathrm{U}\right)$, is characterized (Yang et al., 2017). The coding of circRNAs has not been elucidated in plants, which potentially sheds light on another landscape. 


\section{REGULATORY FUNCTIONS OF PRIMARY MICRORNA-DERIVED PEPTIDES}

Although there are modest pieces of evidence that endogenous peptides can be encoded by ncRNAs in plants, only the regulatory functions of pri-miRNA-derived peptides are well deciphered in available examples. MiRNAs are $~ 22$-nt regulatory elements that inhibit the expression of endogenous genes at both the transcriptional and post-transcriptional levels (Voinnet, 2009). The biogenesis of miRNAs is initialized by the Pol II-dependent transcription of intergenic $M I R$ genes. Mature miRNAs are processed from the much larger pri-miRNA by the Dicerlike RNase III endonucleases (DCLs) complex and assembled into active RNA-induced silencing complex (RISC) through incorporating into ARGONAUTE1 (AGO1) protein (Rogers and Chen, 2013; Yu et al., 2017). The guide strand (miRNA) guides the RISC to bind the target gene via base pairing and mediates gene silencing by target cleavage or translation inhibition (Figure 2; Wang et al., 2019). In addition to producing miRNAs, it was found that the pri-miRNAs can contain short ORFs in the 5' upstream of pre-miRNA, which encode for regulatory peptides, called miPEPs. This coding ability of primiRNAs has first been discovered in Arabidopsis and Medicago truncatula and has since been studied in soybeans, grapes, and even mammalian cells (Lauressergues et al., 2015; Couzigou et al., 2016; Fang et al., 2017; Chen Q. J. et al., 2020; Sharma et al., 2020). The endogenous miPEPs have been detected by Western blotting, demonstrating significant levels of peptide accumulation (Lauressergues et al., 2015; Sharma et al., 2020; Table 1).

The mature miRNA processing mainly finishes in the nucleus and export to the cytoplasm (Wang et al., 2019). Firstly, the pri-miRNAs could be cut by the DCL complex into three parts in the nucleus: (1) the upstream of a precursor of miRNAs (pre-miRNAs), (2) the pre-miRNAs, and (3) the 3' fragments containing poly (A) tail. The pre-miRNAs are further processed into mature miRNA, and the 3' fragments are possibly degraded (Rogers and Chen, 2013). It is also reasonably speculated that the upstream of pre-miRNAs, which possibly contain sORF, is exported to the cytoplasm for guiding peptide translation (Figure 2). For example, the pri-miR171d is mainly accumulated in the nucleus and also slightly detected in the cytoplasm of grape, implying that the coding region of pri-miRNA is possibly transported into the cytoplasm after they are cleaved (Lauressergues et al., 2015; Chen Q. J. et al., 2020). The possibility that the cleaved upstream fragments of the pre-miRNAs are

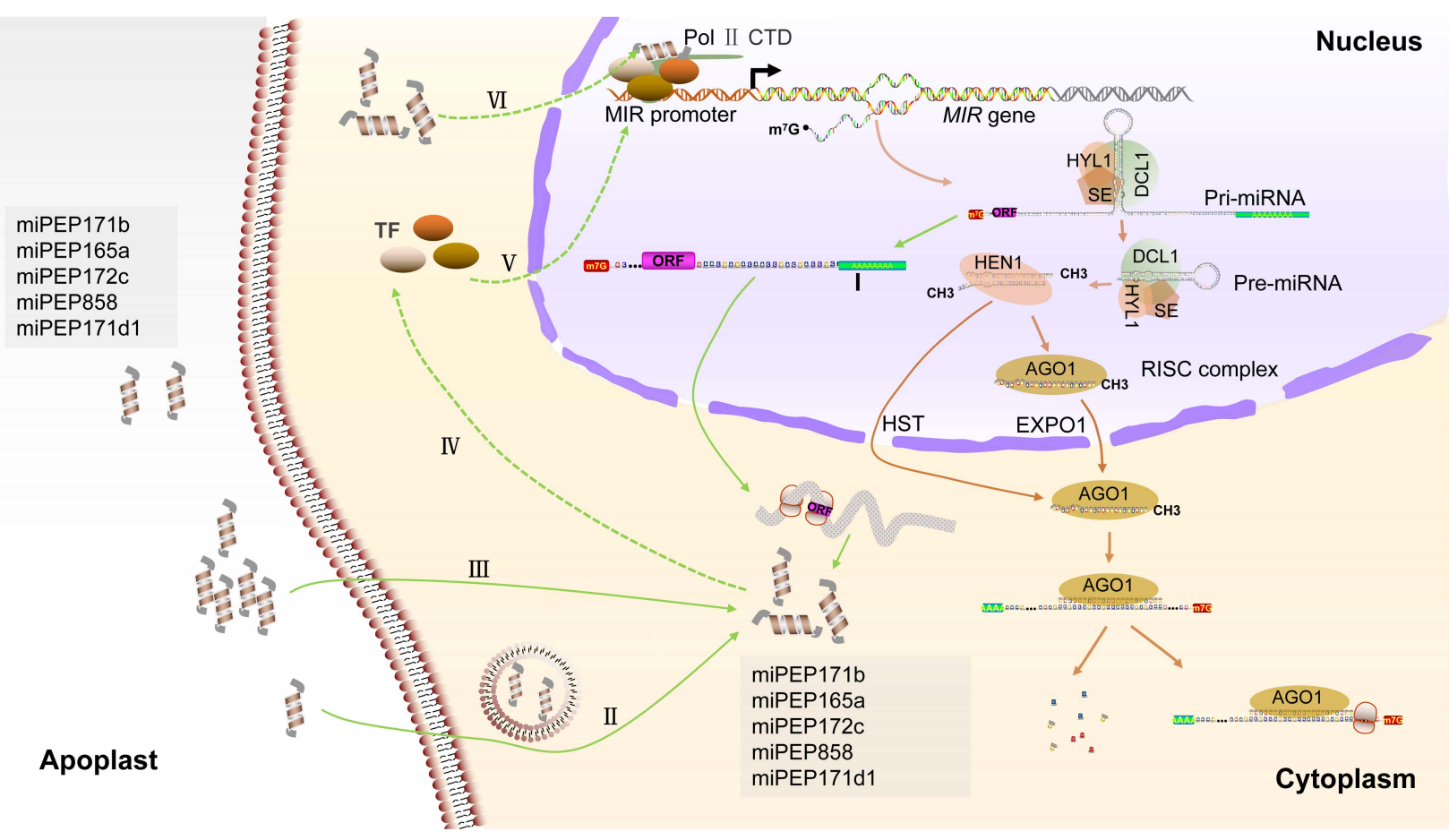

FIGURE 2 | Biogenesis of miRNA and putative regulatory mechanisms of miPEPs in plants. Pri-miRNAs are transcribed by the RNA Pol II from intergenic MIR genes and processed by DCL complex, which is generally composed of dicer-like1 (DCL1), hyponasticleaves1 (HYL1), and serrate (SE). On the one hand, these miRNA/miRNA* duplexes that are methylated by the methyltransferase HUA enhancer1 (HEN1) and assembled into RISC in the nucleus or exported by the HST to cytoplasm for RISC assembling. RISC located in the nucleus can be exported by exportin 1 (EXPO1) to the cytoplasm (Wang et al., 2019). On the other hand, the upstream of pre-miRNA containing short ORF is possibly polyadenylated for preventing degradation (I) and is exported to cytoplasm for guiding miPEPs synthesis. External miPEPs are supposed to internalize into cytoplasm by endocytosis-associated processes (II) and passive diffusion (III) (Ormancey et al., 2020; Sharma et al., 2020). MiPEPs maybe function to transcription factors (TFs), which regulate the transcription of MIR genes (IV and V), resulting in upregulated expression of associated miRNA. Alternatively, miPEPs maybe serve as a part of "Pol II transcriptional complex" and enhance the transcription of MIR genes (VI). 
TABLE 1 | Length and biological function of known miPEPs in planta

\begin{tabular}{|c|c|c|c|c|}
\hline MiPEP name & MiPEP length (a.a.) & Species & Biological function & References \\
\hline miPEP171b & 20 & Medicago truncatula & Regulation of root development & Lauressergues et al., 2015 \\
\hline miPEP169d & NA & Medicago truncatula & NA & Lauressergues et al., 2015 \\
\hline miPEP171e & NA & Medicago truncatula & NA & Lauressergues et al., 2015 \\
\hline MiPEP165a & 18 & Arabidopsis thaliana & $\begin{array}{l}\text { Regulation of root } \\
\text { development, inflorescence } \\
\text { stem, and flowering time }\end{array}$ & Lauressergues et al., 2015; Ormancey et al., 2020 \\
\hline miPEP160b & 24 & Arabidopsis thaliana & NA & Lauressergues et al., 2015 \\
\hline miPEP164a & 37 & Arabidopsis thaliana & NA & Lauressergues et al., 2015 \\
\hline miPEP319a & 50 & Arabidopsis thaliana & NA & Lauressergues et al., 2015 \\
\hline miPEP858a & 44 & Arabidopsis thaliana & $\begin{array}{l}\text { Controlling of flavonoid } \\
\text { biosynthesis and development }\end{array}$ & Sharma et al., 2020 \\
\hline miPEP171c & 10 & Arabidopsis thaliana & Regulation of primary roots & Lauressergues et al., 2015; Chen Q. J. et al., 2020 \\
\hline miPEP171d1 & 7 & Vitis vinifera & $\begin{array}{l}\text { Regulation of adventitious root } \\
\text { formation }\end{array}$ & Chen Q. J. et al., 2020 \\
\hline miPEP172c & 16 & Glycine max & Stimulating nodulation & Couzigou et al., 2016 \\
\hline
\end{tabular}

aNumerous putative miPEPs identified in A. thaliana are presented in a previous report (Lauressergues et al., 2015).

protected by re-polyadenylation is still unknown and requires investigation (Figure 2). What's more, coupling transcription and translation in nuclear are presumed to be an alternative hypothesis (Prasad et al., 2020). The current researches suggest that plant miPEPs may serve as an endogenous peptide to positively amplify the autoregulatory feedback loop of miRNA generation (Ormancey et al., 2020). They are supposed to specifically activate transcription of their corresponding primiRNAs and subsequently to upregulate the expression of mature miRNAs and, meanwhile, to enhance the accumulation of their own levels. The positive regulatory function of miPEPs on MIR transcription in the plant has been investigated. In Arabidopsis, the positive effect of miPEP165a on pri-miR165a accumulation is inhibited by the cordycepin, which is an inhibitor of RNA synthesis (Lauressergues et al., 2015). In Arabidopsis, the promotor of miR858a normally activates the expression of the GUS gene in two reporter lines (PromiR858a:ATG ${ }^{1}$ :GUS and PromiR858a:ORF ${ }^{1}$ GUS), which is fused only the start code or entire ORF encoded miPEP858a. Furthermore, the GUS activity is enhanced by the supplement with synthetic miPEP858a in the media, indicating that the miPEP858a acts on the promotor region for enhancing transcription (Sharma et al., 2020). On the one hand, miPEPs possibly, directly or indirectly, function as a trans-acting factor such as a transcription factor (TF), which positively regulates the transcription of MIR genes (Figure 2). Conventional peptides can regulate TF expression levels in the plant. Root meristem growth factor 1 is a secreted and Tyr sulfated peptide and required to maintain the root stem cell niche and transit amplifying cell proliferation in Arabidopsis. Root meristem growth factor 1 positively regulates the expression levels of PLT, which is a root-specific TF mediating pattern of the root stem cell niche (Matsuzaki et al., 2010). On the other hand, miPEPs may bind one of the subunits of "Pol II transcriptional complexes" or bind to the Pol II, although there are no direct pieces of evidence to support this hypothesis (Figure 2). In plants, peptides can also interact with the subunit of catalyzing enzyme. A representative example is ENOD40 peptides in legumes. Two overlapped ORFs located in $5^{\prime}$ conserved region encode two peptides of 12- and 24-a.a. length residues (peptides A and B); both peptides can specifically bind to nodulin 100 that is a subunit of sucrose synthase (Röhrig et al., 2002). In a word, the strictly regulatory mechanisms of miPEPs remain elusive.

Interestingly, the external application of synthetic miPEPs, which probably do not need additional modification and processing, to plants can produce the same autoregulatory effect. Unlike precursor-derived peptides that usually act as ligand recognized by associated receptors, miPEPs are hypothesized to be internalized by passive diffusion and endocytosis-associated processes (Kachroo et al., 2001; Okuda et al., 2009; Yamaguchi et al., 2010; Oh et al., 2018; Ormancey et al., 2020). In Arabidopsis, fluorescently labeled miPEP165a rapidly penetrates into the whole root during $24 \mathrm{~h}$. Loss of function of genes associated with endocytosis or application of endocytosis inhibitor influences the uptake of miPEP165a in the meristematic zone and differentiation zone (Ormancey et al., 2020). Similar to observations of miPEP165a, miPEP858a can be absorbed by the roots and presence inside the plant cell (Sharma et al., 2020). These results indirectly provide pieces of evidence that miPEPs possibly play a role for regulatory functions within intracellular space rather than be transported into the apoplast (Figure 2).

An investigation of 50 Arabidopsis pri-miRNAs uncovers the presence of at least one putative ORF encoding miPEPs in one pri-miRNA (Lauressergues et al., 2015). These miPEPs have no common signatures, implying that each of these miPEPs is likely specific for their miRNA (Lauressergues et al., 2015). Such a large number of miPEPs form a complex and specific regulatory network that performs different biological functions by positively regulating miRNA expression level. So far, the biological function of numerable miPEPs has been deciphered. MiPEP171b and MiPEP165a are 20-a.a. and 18-a.a. peptides produced by $M$. truncatula and Arabidopsis, respectively. Overexpression and exogenous supplement of these peptides specifically trigger the accumulation of $m i R 171 b$ and $m i R 165 a$, resulting in decreased lateral root formation and stimulation of 
main root growth (Lauressergues et al., 2015). Watering plants with synthetic miPEP172c increases nodule number in soybean (Couzigou et al., 2016). In grape, an exogenous supplement of vvimiPEP171d1 can promote adventitious root development by enhancing the expression of $v v i-M I R 171 d$ (Chen Q. J. et al., 2020). MiPEP858a is a 44-a.a. peptide encoded by the first ORF (135 bp) located upstream in the pre-miR858a sequence in Arabidopsis. The endogenous miPEP858a is $\sim 6 \mathrm{kDa}$ in molecular weight (Sharma et al., 2020). MiPEP858a controls flavonoid biosynthesis and plant development by regulating the expression of genes involved in the phenylpropanoid pathway and auxin signaling (Sharma et al., 2020). Although the regulatory functions of several miPEPs have been experimentally validated in different plant species, some key questions remain to be answered. Is miPEPs specific for upregulation of their corresponding primiRNAs, and if so, how is it achieved? After all, only a few miRNAs have been used to detect activation specificity (Lauressergues et al., 2015). In grape, the expression level of some miRNAs genes such as vvi-MIR160c, vviMIR171a, and $v v i$ $M I R 171 i$ even reduces when grape tissue culture plantlets are treated by synthetic vvi-miPEP171d1, even if it is not definitely clear whether this decrease is caused by the incubation period (Chen Q. J. et al., 2020). In fact, small peptides encoded by lncRNAs produce either inhibitory or stimulatory effects on their target genes in mammals (Anderson et al., 2015; Nelson et al., 2016). Therefore, whether miPEPs exert a negative effect on their corresponding miRNAs or other miRNAs remains to be shown.

\section{PERSPECTIVE}

Peptides are regulatory molecules that have received great attention over recent years. In particular, different types of peptides identified from several species were found to be enriched in the peptidome of plants (Tavormina et al., 2015; Olsson et al., 2019; Takahashi et al., 2019). In addition to the conventional peptides derived from the precursor processing and short ORFs, pieces of evidence are emerging for the presence and function of non-conventional peptides translated from 5' UTR or 3' UTR of transcripts and currently defined as ncRNAs (Wang et al., 2020). The function of peptides is diverse, and they are found to be involved in development, growth, and reproduction, senescence and cell death, nutrients balance and nodulation, and biotic and abiotic stress responses. In the past few years, numerous miPEPs derived from pri-miRNAs have been experimentally identified and their function suggested by overexpression studies in different species, implying that coding of pri-miRNAs is ubiquitous among different plant species. Existing pieces of evidence support the regulatory ability of miPEPs in directing an increase in the level of their associated

\section{REFERENCES}

Anderson, D. M., Anderson, K. M., Chang, C. L., Makarewich, C. A., Nelson, B. R., McAnally, J. R., et al. (2015). A micropeptide encoded by a putative long noncoding RNA regulates muscle performance. Cell 160, 595-606. doi: 10.1016/j.cell.2015.01.009
miRNA by enhancing the transcription of pri-miRNAs. Still, several questions remain to be addressed. (1) MiPEPs synthesis and miRNA processing occur in two independent regions of pri-miRNAs; how does coordination occur between the translation in the cytoplasm and the maturation of miRNA in the nucleus? One putative mechanism is that the $5^{\prime}$ upstream of pri-miRNA is transported into the cytoplasm for guiding miPEP synthesis after releasing from the dicing complex and re-polyadenylation (Figure 2). (2) How do miPEPs specifically enhance transcription of the MIR gene? An analysis of 50 Arabidopsis miPEPs uncovered no common structural pattern among them, suggesting that each miPEP probably has a specific regulatory function (Lauressergues et al., 2015). Whether miPEPs directly bind Pol II or trans-acting factors such as TFs remains to be further investigated. (3) Regarding the exogenous application of miPEPs, overexpression and CRISPR-Cas9-editing are required for assessing the biological functions. It is not clear that post-translational modifications of miPEPs can enhance the activity of miPEPs.

In summary, most characterized peptides to date are hypothesized to act as a ligand to mediate plant intercellular communication and response. The identification of peptides that are translated from the transcript of currently defined ncRNAs enriches the plants' peptidome. Particularly, miPEP identification uncovers the dual function of pri-miRNAs combing with coding and non-coding ability. Dissecting the biosynthesis and regulatory mechanism of miPEPs will reveal another miRNAdependent gene regulation network.

\section{AUTHOR CONTRIBUTIONS}

YR integrated the manuscript and drafted the figures. YS, $\mathrm{LZ}, \mathrm{DG}$, and $\mathrm{JH}$ retrieved and collected the references about miRNA biogenesis, translation of circRNAs, and the translation of IncRNA. LW, SS, WX, and CZ retrieved and collected the literatures about canonical peptides biogenesis, and biological functions. AL and SW revised the manuscript. CM conceived the idea and revised the manuscript. All authors contributed to the article and approved the submitted version.

\section{FUNDING}

This work was supported by the Shanghai municipal key task projects of "Prospering Agriculture by Science and Technology Plan" (Grant No. 2020-02-08-00-08-F01458), the National Natural Science Foundation of China (Grant No. 31972383), and the Shanghai Municipal Commission for Science and Technology (Grant No. 19ZR1428000).

Andrews, S. J., and Rothnagel, J. A. (2014). Emerging evidence for functional peptides encoded by short open reading frames. Nat. Rev. Genet. 15, 193-204. doi: $10.1038 / \mathrm{nrg} 3520$

Casson, S. A., Chilley, P. M., Topping, J. F., Evans, I. M., Souter, M. A., and Lindsey, K. (2002). The POLARIS gene of Arabidopsis encodes a predicted peptide required for correct root growth and 
leaf vascular patterning. Plant Cell 14, 1705-1721. doi: 10.1105/tpc.00 2618

Chekanova, J. A. (2015). Long non-coding RNAs and their functions in plants. Curr. Opin. Plant Biol. 27, 207-216. doi: 10.1016/j.pbi.2015.08.003

Chen, J., Brunner, A. D., Cogan, J. Z., Nuñez, J. K., Fields, A. P., Adamson, B., et al. (2020). Pervasive functional translation of noncanonical human open reading frames. Science 367, 1140-1146. doi: 10.1126/science.aay0262

Chen, Q. J., Deng, B. H., Gao, J., Zhao, Z. Y., Chen, Z. L., Song, S. R., et al. (2020). A miRNA-encoded small peptide, vvi-miPEP171d1, regulates adventitious root formation. Plant Physiol. 183, 656-670. doi: 10.1104/pp.20.00197

Conn, V. M., Hugouvieux, V., Nayak, A., Conos, S. A., Capovilla, G., Cildir, G., et al. (2017). A circRNA from SEPALLATA3 regulates splicing of its cognate mRNA through R-loop formation. Nat. Plants 3:17053. doi: 10.1038/nplants.2017.53

Couzigou, J. M., André, O., Guillotin, B., Alexandre, M., and Combier, J. P. (2016). Use of microRNA-encoded peptide miPEP172c to stimulate nodulation in soybean. New Phytol. 211, 379-381. doi: 10.1111/nph.13991

Diallo, L. H., Tatin, F., David, F., Godet, A. C., Zamora, A., Prats, A. C., et al. (2019). How are circRNAs translated by non-canonical initiation mechanisms? Biochimie 164, 45-52. doi: 10.1016/j.biochi.2019.06.015

Dubois, M., Van den Broeck, L., and Inze, D. (2018). The pivotal role of ethylene in plant growth. Trends Plant Sci. 23, 311-323. doi: 10.1016/j.tplants.2018.01.003

Fang, J., Morsalin, S., Rao, V., and Reddy, E. S. (2017). Decoding of non-coding DNA and non-coding RNA: pri-micro RNA-encoded novel peptides regulate migration of cancer cells. J. Pharm. Pharmacol. 3, 23-27. doi: 10.1166/jpsp.2017. 1070

Gao, Z., Li, J., Luo, M., Li, H., Chen, Q., Wang, L., et al. (2019). Characterization and cloning of grape circular RNAs identified the cold resistance-related VvcircATS1. Plant Physiol. 180, 966-985. doi: 10.1104/pp.18.01331

Guttman, M., Russell, P., Ingolia, N. T., Weissman, J. S., and Lander, E. S. (2013). Ribosome profiling provides evidence that large noncoding RNAs do not encode proteins. Cell 154, 240-251. doi: 10.1016/j.cell.2013.06.009

Hanada, K., Higuchi-Takeuchi, M., Okamoto, M., Yoshizumi, T., Shimizu, M., Nakaminami, K., et al. (2013). Small open reading frames associated with morphogenesis are hidden in plant genomes. Proc. Natl. Acad. Sci. U.S.A. 110, 2395-2400. doi: 10.1073/pnas.1213958110

Hsu, P. Y., and Benfey, P. N. (2018). Small but mighty: functional peptides encoded by small ORFs in plants. Proteomics 18:e1700038. doi: 10.1002/pmic.201700038

Huang, J. Z., Chen, M., Chen, D., Gao, X. C., Zhu, S., Huang, H., et al. (2017). A peptide encoded by a putative lncRNA HOXB-AS3 suppresses colon cancer growth. Mol. Cell 68, 171-184.e176. doi: 10.1016/j.molcel.2017.09.015

Huffaker, A., Dafoe, N. J., and Schmelz, E. A. (2011). ZmPep1, an ortholog of Arabidopsis elicitor peptide 1, regulates maize innate immunity and enhances disease resistance. Plant Physiol. 155, 1325-1338. doi: 10.1104/pp.110. 166710

Huffaker, A., Pearce, G., and Ryan, C. A. (2006). An endogenous peptide signal in Arabidopsis activates componenets of the innate immune response. Proc. Natl. Acad. Sci. U.S.A. 103, 10098-10103. doi: 10.1073/pnas.0603727103

Huffaker, A., and Ryan, C. A. (2007). Endogenous peptide defense signals in Arabidopsis differentially amplify sigbaling for the innate immnue response. Proc. Natl. Acad. Sci. U.S.A. 104, 10732-10736. doi: 10.1073/pnas.0703343104

Ingolia, N. T., Brar, G. A., Rouskin, S., McGeachy, A. M., and Weissman, J. S. (2012). The ribosome profiling strategy for monitoring translation in vivo by deep sequencing of ribosome-protected mRNA fragments. Nat. Protoc. 7, 1534-1550. doi: 10.1038/nprot.2012.086

Julkowska, M. (2020). Small but powerful: microRNA-derived peptides promote grape adventitious root formation. Plant Physiol. 183, 429-430. doi: 10.1104/ pp. 20.00515

Kachroo, A., Schopfer, C. R., Nasrallah, M. E., and Nasrallah, J. B. (2001). Allelespecific receptor-ligand interactions in Brassica self-incompatibility. Science 293, 1824-1826. doi: 10.1126/science.1062509

Kieber, J. J., and Schaller, G. E. (2018). Cytokinin signaling in plant development. Development 145:dev149344. doi: 10.1242/dev.149344

Kurihara, Y. (2020). uORF shuffling fine-tunes gene expression at a deep level of the process. Plants 9:608. doi: 10.3390/plants9050608

Laing, W. A., Martinez-Sanchez, M., Wright, M. A., Bulley, S. M., Brewster, D., Dare, A. P., et al. (2015). An upstream open reading frame is essential for feedback regulation of ascorbate biosynthesis in Arabidopsis. Plant Cell 27, 772-786. doi: 10.1105/tpc.114.133777
Lauressergues, D., Couzigou, J. M., Clemente, H. S., Martinez, Y., Dunand, C., Becard, G., et al. (2015). Primary transcripts of microRNAs encode regulatory peptides. Nature 520, 90-93. doi: 10.1038/nature14346

Legnini, I., Di Timoteo, G., Rossi, F., Morlando, M., Briganti, F., Sthandier, O., et al. (2017). Circ-ZNF609 is a circular RNA that can be translated and functions in myogenesis. Mol. Cell 66, 22-37 e29. doi: 10.1016/j.molcel.2017. 02.017

Li, X., Yang, L., and Chen, L. L. (2018). The biogenesis, functions, and challenges of circular RNAs. Mol. Cell 71, 428-442. doi: 10.1016/j.molcel.2018. 06.034

Liu, H., Zhou, X., Yuan, M., Zhou, S., Huang, Y. E., Hou, F., et al. (2020). ncEP: a manually curated database for experimentally validated ncRNA-encoded proteins or peptides. J. Mol. Biol. 432, 3364-3368. doi: 10.1016/j.jmb.2020.02. 022

Lv, S., Pan, L., and Wang, G. (2016). Commentary: primary transcripts of microRNAs encode regulatory peptides. Front. Plant Sci. 7:1436. doi: 10.3389/ fpls.2016.01436

Matsubayashi, Y. (2014). Posttranslationally modified small-peptide signals in plants. Annu. Rev. Plant Biol. 65, 385-413. doi: 10.1146/annurev-arplant050312-120122

Matsuzaki, Y., Ogawa-Ohnishi, M., Mori, A., and Matsubayashi, Y. (2010). Secreted peptide signals required for maintenance of root stem cell niche in Arabidopsis. Science 329, 1065-1067. doi: 10.1126/science.1191132

Nakaminami, K., Okamoto, M., Higuchi-Takeuchi, M., Yoshizumi, T., Yamaguchi, Y., Fukao, Y., et al. (2018). AtPep3 is a hormone-like peptide that plays a role in the salinity stress tolerance of plants. Proc. Natl. Acad. Sci. U.S.A. 115, 5810-5815. doi: 10.1073/pnas.1719491115

Nelson, B. R., Makarewich, C. A., Anderson, D. M., and Winders, B. R. (2016). A peptide encoded by transcript annotated as long noncoding RNA enhances SERCA activity in muscle. Science 351, 271-275. doi: 10.1126/science.aad4076

Oh, E., Seo, P. J., and Kim, J. (2018). Signaling peptides and receptors coordinating plant root development. Trends Plant Sci. 23, 337-351. doi: 10.1016/j.tplants. 2017.12.007

Ohkubo, Y., Tanaka, M., Tabata, R., Ogawa-Ohnishi, M., and Matsubayashi, Y. (2017). Shoot-to-root mobile polypeptides involved in systemic regulation of nitrogen acquisition. Nat. Plants 3:17029. doi: 10.1038/nplants.2017.29

Okamoto, S., Tabata, R., and Matsubayashi, Y. (2016). Long-distance peptide signaling essential for nutrient homeostasis in plants. Curr. Opin. Plant. Biol. 34, 35-40. doi: 10.1016/j.pbi.2016.07.009

Okuda, S., Tsutsui, H., Shiina, K., Sprunck, S., Takeuchi, H., Yui, R., et al. (2009). Defensin-like polypeptide LUREs are pollen tube attractants secreted from synergid cells. Nature 458, 357-361. doi: 10.1038/nature07882

Olsson, V., Joos, L., Zhu, S., Gevaert, K., Butenko, M. A., and De Smet, I. (2019). Look closely, the beautiful may be small: precursor-derived peptides in plants. Annu. Rev. Plant Biol. 70, 153-186. doi: 10.1146/annurev-arplant-042817040413

Ormancey, M., Le Ru, A., Duboe, C., Jin, H., Thuleau, P., Plaza, S., et al. (2020). Internalization of miPEP165a into Arabidopsis roots depends on both passive diffusion and endocytosis-associated processes. Int. J. Mol. Sci. 21:2266. doi: $10.3390 /$ ijms 21072266

Pearce, G., Strydom, D., JohnsON, S., and Ryan, C. A. (1991). A polypeptide from tomato Leaves induces wound-inducible proteinase inhibitor proteins. Science 253, 895-897. doi: 10.1126/science.253.5022.895

Prasad, A., Sharma, N., and Prasad, M. (2020). Noncoding but coding: pri-miRNA into the action. Trends Plant Sci. S1360-1385, 30381-30382. doi: 10.1016/j. tplants.2020.12.004

Qian, P., Song, W., Yokoo, T., Minobe, A., Wang, G., Ishida, T., et al. (2018). The CLE9/10 secretory peptide regulates stomatal and vascular development through distinct receptors. Nat. Plants 4, 1071-1081. doi: 10.1038/s41477-0180317-4

Qu, L. J., Li, L., Lan, Z., and Dresselhaus, T. (2015). Peptide signalling during the pollen tube journey and double fertilization. J. Exp. Bot. 66, 5139-5150. doi: 10.1093/jxb/erv275

Ribone, P. A., Capella, M., Arce, A. L., and Chan, R. L. (2017). A uORF represses the transcription factor AtHB1 in aerial tissues to avoid a deleterious phenotype. Plant Physiol. 175, 1238-1253. doi: 10.1104/pp.17.01060

Rogers, K., and Chen, X. (2013). Biogenesis, turnover, and mode of action of plant microRNAs. Plant Cell 25, 2383-2399. doi: 10.1105/tpc.113.113159 
Röhrig, H., Schmidt, J., Miklashevichs, E., Schell, J., and John, M. (2002). Soybean ENOD40 encodes two peptides that bind to sucrose synthase. Proc. Natl. Acad. Sci. U.S.A. 99, 1915-1920. doi: 10.1073/pnas.022664799

Ruiz-Orera, J., Messeguer, X., Subirana, J. A., and Alba, M. M. (2014). Long noncoding RNAs as a source of new peptides. ELife 3:e03523. doi: 10.7554/eLife. 03523

Sharma, A., Badola, P. K., Bhatia, C., Sharma, D., and Trivedi, P. K. (2020). Primary transcript of miR858 encodes regulatory peptide and controls flavonoid biosynthesis and development in Arabidopsis. Nat. Plants 6, 1262-1274. doi: 10.1038/s41477-020-00769-x

Shi, Y., Jia, X., and Xu, J. (2020). The new function of circRNA: translation. Clin. Transl. Oncol. 22, 2162-2169. doi: 10.1007/s12094-020-02371-1

Takahashi, F., Hanada, K., Kondo, T., and Shinozaki, K. (2019). Hormone-like peptides and small coding genes in plant stress signaling and development. Curr. Opin. Plant Biol. 51, 88-95. doi: 10.1016/j.pbi.2019.05.011

Takahashi, F., Suzuki, T., Osakabe, Y., Betsuyaku, S., Kondo, Y., Dohmae, N., et al. (2018). A small peptide modulates stomatal control via abscisic acid in long-distance signalling. Nature 556, 235-238. doi: 10.1038/s41586-018-00092

Tavormina, P., De Coninck, B., Nikonorova, N., De Smet, I., and Cammue, B. P. (2015). The plant peptidome: an expanding repertoire of structural features and biological functions. Plant Cell 27, 2095-2118. doi: 10.1105/tpc.15.00440

van der Horst, S., Snel, B., Hanson, J., and Smeekens, S. (2019). Novel pipeline identifies new upstream ORFs and non-AUG initiating main ORFs with conserved amino acid sequences in the 5' leader of mRNAs in Arabidopsis thaliana. RNA 25, 292-304. doi: 10.1261/rna.067983.118

van Heesch, S., van Iterson, M., Jacobi, J., Boymans, S., Essers, P. B., de Bruijn, E., et al. (2014). Extensive localization of long noncoding RNAs to the cytosol and mono- and polyribosomal complexes. Genome Biol. 15:R6. doi: 10.1186/gb2014-15-1-r6

Voinnet, O. (2009). Origin, biogenesis, and activity of plant microRNAs. Cell 136, 669-687. doi: 10.1016/j.cell.2009.01.046

von Arnim, A. G., Jia, Q., and Vaughn, J. N. (2014). Regulation of plant translation by upstream open reading frames. Plant Sci. 214, 1-12. doi: 10.1016/j.plantsci. 2013.09.006

Wang, J., Mei, J., and Ren, G. (2019). Plant microRNAs: biogenesis, homeostasis, and degradation. Front. Plant Sci. 10:360. doi: 10.3389/fpls.2019.00360

Wang, L., and Wang, J. W. (2015). Coding function for non-coding RNA in plants-insights from miRNA encoded peptide (miPEP). Sci. China Life Sci. 58, 503-505. doi: 10.1007/s11427-015-4854-z
Wang, S., Tian, L., Liu, H., Li, X., Zhang, J., Chen, X., et al. (2020). Large-scale discovery of non-conventional peptides in maize and Arabidopsis through an integrated peptidogenomic pipeline. Mol. Plant 13, 1078-1093. doi: 10.1016/j. molp.2020.05.012

Wang, Y., and Wang, Z. (2015). Efficient backsplicing produces translatable circular mRNAs. RNA 21, 172-179. doi: 10.1261/rna.048272.114

Wu, Q., Wright, M., Gogol, M. M., Bradford, W. D., Zhang, N., and Bazzini, A. A. (2020). Translation of small downstream ORFs enhances translation of canonical main open reading frames. EMBO J. 39:e104763. doi: 10.15252/embj. 2020104763

Yamaguchi, Y., Huffaker, A., Bryan, A. C., Tax, F. E., and Ryan, C. A. (2010). PEPR2 is a second receptor for the Pep1 and Pep2 peptides and contributes to defense responses in Arabidopsis. Plant Cell 22, 508-522. doi: 10.1105/tpc.109.068874

Yamaguchi, Y., Pearce, G., and Ryan, C. A. (2006). The cell surface leucine-rich repeat receptor for AtPep1, an endogenous peptide elicitor in Arabidopsis, is functional in transgenic tobacco cells. Proc. Natl. Acad. Sci. U.S.A. 103, 10104-10109. doi: 10.1073/pnas.0603729103

Yamaguchi, Y. L., Ishida, T., and Sawa, S. (2016). CLE peptides and their signaling pathways in plant development. J. Exp. Bot. 67, 4813-4826. doi: 10.1093/jxb/ erw208

Yang, Y., Fan, X., Mao, M., Song, X., Wu, P., Zhang, Y., et al. (2017). Extensive translation of circular RNAs driven by $\mathrm{N}^{6}$-methyladenosine. Cell Res. 27, 626-641. doi: 10.1038/cr.2017.31

Yu, Y., Jia, T., and Chen, X. (2017). The 'how' and 'where' of plant microRNAs. New Phytol. 216, 1002-1017. doi: 10.1111/nph.14834

Zhang, H., Si, X., Ji, X., Fan, R., Liu, J., and Chen, K. (2018). Genome editing of upstream open reading frames enables translational control in plants. Nat. Biotechnol. 36, 894-898. doi: 10.1038/nbt.4202

Conflict of Interest: The authors declare that the research was conducted in the absence of any commercial or financial relationships that could be construed as a potential conflict of interest.

Copyright (C) 2021 Ren, Song, Zhang, Guo, He, Wang, Song, Xu, Zhang, Lers, Ma and Wang. This is an open-access article distributed under the terms of the Creative Commons Attribution License (CC BY). The use, distribution or reproduction in other forums is permitted, provided the original author(s) and the copyright owner(s) are credited and that the original publication in this journal is cited, in accordance with accepted academic practice. No use, distribution or reproduction is permitted which does not comply with these terms. 